\title{
Testing fractional order of long memory processes: A Monte Carlo study
}

\author{
Laurent FERRARA, D Dominique GUEGAN † Zhiping LU $\ddagger$
}

February 20, 2008

\begin{abstract}
Testing the fractionally integrated order of seasonal and non-seasonal unit roots is quite important for the economic and financial time series modelling. In this paper, Robinson test (1994) is applied to various well-known long memory models. Via Monte Carlo experiments, we study and compare the performances of this test using several sample sizes.
\end{abstract}

Keywords: Long memory processes - Test - Monte Carlo Simulations.

JEL: C12 - C15 - C22.

\section{Introduction}

Usefulness of fractionally integrated processes has been pointed out over the recent years in order to take various strong persistence effects into modelling. Macroeconomics works focusing on the modelling and forecasting of economic activity time series have made an extensive use of the fractional alternative through long memory processes. For example, Carlin and Dempster (1989) consider monthly unemployment rate of US males; Porter Hudak (1990) deals

*Banque de France, DGEI-DAMEP and CES Université Paris 1 Panthéon-Sorbonne, laurent.ferrara@banque-france.fr

†Paris School of Economics, CES-MSE, Université Paris 1 Panthéon-Sorbonne, 106-112 bd de l'Hôpital, 75013, Paris, FRANCE, dguegan@univ-paris1.fr

${ }^{\ddagger}$ Department of Mathematics, East China Normal University, 200241, P.R. China; Ecole Normale Supérieure de Cachan, 61, Avenue du Président Wilson, 94235, Cachan, camping9@gmail.com 
with the US money supply and monetary aggregates and Ray (1993) proposes models for monthly IBM revenue data. Monthly UK inflation rates have been considered by Franses and Ooms (1997), Arteche and Robinson (2000) and Arteche (2003). Other applications also deal with time series on consumer goods (Darné, Guiraud and Terraza, 2004), public transportation (Ferrara and Guégan, 2000), exchange rates (Ferrara and Guégan, 2001a), spot prices (Ferrara and Guégan, 2001b) or electricity prices (Diongue and Guégan, 2004).

For all those applications, a specific fractionally integrated process has been proposed by the researchers. Generally the choice of the process corresponds to a specific problematic and no comparison has been carried out with different types of long memory processes. For example, some papers use the classical fractionally integrated process, introduced by Granger and Joyeux (1980) and Hosking (1981), while others focus on generalized long memory processes or seasonal long memory processes dedicated to take cyclical or seasonal components with persistence into account. In this paper, we consider the general class of fractionally integrated processes $\left(X_{t}\right)_{t}$ mean zero, referred to as Seasonal/Cyclical Long Memory (SCLM) processes, defined by the following equation:

$$
F(B) X_{t}=(I-B)^{d_{0}+\theta_{0}} \prod_{i=1}^{k-1}\left(I-2 \nu_{i} B+B^{2}\right)^{d_{i}+\theta_{i}}(I+B)^{d_{k}+\theta_{k}} X_{t}=\varepsilon_{t}
$$

where $B$ is the backshift operator. For $i=1, \cdots, k-1, \nu_{i}=\cos \lambda_{i}, \lambda_{i}$ being any frequency between 0 and $\pi$. For $i=0,1, \cdots k, \theta_{i}$ belongs to $[-1,1]$ and, $\left|d_{i}\right|<1 / 2$ implying that the spectral density is unbounded in $\lambda_{i}$. Moreover, $\left(\varepsilon_{t}\right)_{t}$ is an innovation process to be specified. This model has been first discussed by Robinson (1994) in order to test whether the data stem from a stationary or a non-stationary process, under uncorrelated and weakcorrelated innovations $\left(\varepsilon_{t}\right)_{t}$. The process described by equation (1) nests all the specific long memory processes generally used in applications.

From a practical point of view, before implementing a fractional process on real data, it is warmly recommended to carry out a statistical test to show evidence of persistence in the data. In this respect, the test of Robinson (1994) has been proven to be very useful for testing stationarity of many SCLM processes, Gil-Alana (2001, 2006). This test also permits to test the integration order at various frequencies and does not require the estimation of long memory parameters since the series have a short-memory behaviour under the null hypothesis. Now, this test can also be used to test the de- 
gree of persistence of the memory parameter using the null of Robinson test. However, one of the major drawback in empirical macroeconomics is the rather small amount of data available to the practitioner. For example in the industrialized countries, the broadest measure of economic activity released by the quarterly national accounts of the statistical institutes, namely GDP, is generally available only since 1970 . Thus, in most of the cases, less than 160 data points are available to lead the analysis on a quarterly basis. In this respect, it appears crucial to study the finite sample behaviour of the statistical procedures carried out at each step of the statistical analysis. In this paper, we propose a simulation experiment to determine the possible application of the Robinson (1994) test for finite samples. Indeed, in his paper the results are proved in an asymptotic setting and we need to know how the test works empirically. We study the convergence of the test according to the fractional process used to generate simulated data. We investigate this rate of convergence whatever the innovation process is uncorrelated or weakly correlated.

The paper is organized as follows. In Section two we specify the SCLM processes on which we apply the Robinson test, which is in turn detailed in Section three. Section four is devoted to the results of the simulation experiments. Section five concludes.

\section{Some Seasonal Fractional Models}

From model (1), assuming that $\theta_{i}=0, i=0, \cdots, k$ we can derive in a stationary setting a lot of models whose interest in macroeconomics is recognized. To adjust these models on real data sets, it is fundamental to detect long memory behavior through statistical tests. Here, we investigate Robinson test. The properties of this test have been proved in an asymptotic setting. Working with macro-economics data sets significate that we have very small quartely or monthly data sets. Very often the sample size is around 150 points and less than 1000 points, thus it is important to know the accuracy of this test for a finite sample size. In Section four we provide the rate of convergence for Robinson's test using Monte Carlo simulations. We specify now the models on which we work deriving from the expression (1):

- if $d_{i}=0$, and $\theta_{i}=0, i=1, \cdots, k$, we get the $\mathrm{FI}(d)$ (Fractionally Integrated) process if $\left(\varepsilon_{t}\right)_{t}$ is a white noise:

$$
(I-B)^{d} X_{t}=\varepsilon_{t},
$$


proposed by Granger and Joyeux (1980) and Hosking (1981). If we assume that $\left(\varepsilon_{t}\right)_{t}$ follows a GARCH noise, we get the FIGARCH model, (fractionally integrated and GARCH), Baillie, Bollerslev and Mikkelsen (1996). This class of models permits to take into account the existence of an infinite cycle, as well as the spectral density's typical shape of macroeconomics data, namely an explosion for the very low frequencies.

- In order to model a fixed seasonal periodicity $s$, supposed to be even, we generally use the following representation:

$$
\left(I-B^{s}\right)^{d} X_{t}=\varepsilon_{t}
$$

For instance, if $s=4$, the expression (3) becomes:

$$
\left(I-B^{4}\right)^{d} X_{t}=(I-B)^{d}\left(I+B^{2}\right)^{d}(I+B)^{d} X_{t}=\varepsilon_{t} .
$$

This filter was introduced by Porter-Hudak (1990). It is called the rigid filter and can be regarded as the particular case of Hassler's flexible filter (1994). It is motivated by factorizing $I-B^{4}$ according to its unit roots. This filter allows to model stationary fractional seasonalities. This representation is useful for quartely data sets; if we work with monthly data, we consider the same model using $s=12$.

- It may happens that we observe an explosion at the zero frequency as well as at any frequency between $] 0, \pi]$. This means that an infinite cycle is mixed with another seasonality. In that case, we use the following model for $\left(X_{t}\right)_{t}$ :

$$
(I-B)^{d_{1}}\left(I-B^{s}\right)^{d_{2}} X_{t}=\varepsilon_{t} .
$$

This model was introduced by Porter-Hudak (1990). The parameter $d_{1}$ corresponds to the persistence associated to the infinite cycle and $d_{2}$ is the persistence associated to the fixed seasonality.

- In presence of explosions at $k$ frequencies in the spectral density, we use a model characterizing these $k$ persistent periodicities. It is the $k$-factor Gegenbauer process given by:

$$
\prod_{i=1}^{k}\left(I-2 \nu_{i} B+B^{2}\right)^{d_{i}} X_{t}=\varepsilon_{t},
$$

with, for $i=1, \ldots, k, \nu_{i}=\cos \left(\lambda_{i}\right)$, the frequencies $\lambda_{i}=\cos ^{-1}\left(\nu_{i}\right)$ being the Gegenbauer frequencies or the G-frequencies. When $\left(\varepsilon_{t}\right)_{t}$ is a white noise, this model was introduced by Giraitis and Leipus (1995), and Woodward, Cheng and Gray (1998). When $\left(\varepsilon_{t}\right)_{t}$ follows a GARCH process, the extension was introduced by Guégan (2000, 2003). 
- Inside the spectral density, we can observe $k$ explosions as well as an explosion at the zero frequency, then the previous model becomes:

$$
(I-B)^{d} \prod_{i=1}^{k}\left(I-2 \nu_{i} B+B^{2}\right)^{d_{i}} X_{t}=\varepsilon_{t} .
$$

In Section four, we applied the Robinson test to these models in order to assess its accuracy for various sample sizes.

\section{The Robinson test, 1994}

In this section we briefly describe Robinson test (1994) which is a Lagrange Multiplier test for testing unit roots and other fractionally hypotheses when the roots are located at any frequency inside the interval $[0, \pi]$. The test is derived via the score principle and its asymptotic critical values obey the Chi-squared distribution. Let $\left(Y_{t}\right)_{t}$ be a stochastic process, then we assume that

$$
Y_{t}=\beta^{\prime} Z_{t}+X_{t},
$$

where $Z_{t}$ a $k \times 1$ vector which is observable, $\beta$ an unknown $k \times 1$ vector, and $X_{t}$ a process which follows (1). In the rest of the paper, we assume that $\beta=0$ and $\left(\varepsilon_{t}\right)_{t}$ is a strong white noise or a $\operatorname{GARCH}(1,1)$ noise.

Robinson (1994) worked with the general model (1) for a fixed $d$ and test the assumption

$$
\begin{gathered}
H_{0}: \theta=\left(\theta_{0}, \cdots, \theta_{k}\right)^{\prime}=0, \\
H_{a}: \theta \neq 0 .
\end{gathered}
$$

The test statistic is equal to:

$$
\tilde{R}=\frac{T}{\tilde{\sigma}^{4}} \frac{\tilde{a}^{2}}{\tilde{A}}
$$

where $\mathrm{T}$ is the length of the raw time series and

$$
\tilde{\sigma}^{2}=\frac{2 \pi}{T} \sum_{j}^{*} I_{\tilde{\varepsilon}}\left(\lambda_{j}\right) .
$$

$I_{\tilde{\varepsilon}}\left(\lambda_{j}\right)$ being the periodogram of $\tilde{\varepsilon}_{t}$ with $\tilde{\varepsilon}_{t}=F(B) Y_{t}, F(B)$ is given in equation (1). Moreover:

$$
\tilde{A}=\frac{2}{T} \sum_{j}^{*} \psi\left(\lambda_{j}\right) \cdot \psi\left(\lambda_{j}\right)^{\prime}
$$


and

$$
\tilde{a}^{2}=\frac{-2 \pi}{T} \sum_{j}^{*} \psi\left(\lambda_{j}\right) I\left(\lambda_{j}\right)
$$

where $\sum_{j}^{*}$ is the sum over $\lambda_{j}=\frac{2 \pi j}{T} \in M=\{\lambda:-\pi<\lambda<\pi, \lambda \notin$ $\left.\left(\rho_{l}-\eta, \rho_{l}+\eta\right)\right\}$ such that $\rho_{l}$ are the distinct poles of $\psi(\lambda)$ on $(-\pi, \pi], \eta$ is a given positive constant. Finally

$$
\psi\left(\lambda_{j}\right)=\left(\psi_{l}\left(\lambda_{j}\right)\right)
$$

with

$\psi_{l}\left(\lambda_{j}\right)=\delta_{0 l} \log \left|2 \sin \frac{1}{2} \lambda_{j}\right|+\delta_{k l} \log \left(2 \cos \frac{1}{2} \lambda_{j}\right)+\sum_{i=1}^{k} \delta_{i l} \log \left(\left|2\left(\cos \left(\lambda_{j}\right)-\cos \omega_{i}\right)\right|\right.$,

for $l=0,1, \cdots, k$, where $\delta_{i l}=1$ if $i=l$ and 0 otherwise.

Under stationary conditions, Robinson (1994) establishes that

$$
\tilde{R} \rightarrow_{d} \chi_{k+1}^{2}
$$

where $k+1=\operatorname{dim}(\theta)$. If $\chi_{k+1}^{2}$ represents the $\chi^{2}$ distribution with $k+1$ degrees of freedom then $\chi_{k+1, \alpha}^{2}$ represents a quantile for a given level $\alpha$. As soon as $\tilde{R}>\chi_{k+1, \alpha}^{2}$, we reject $H_{0}$, with risk $\alpha$.

Under the null, the test chooses the best long memory parameter, which corresponds to the greatest $p$-value of the Chi-square test. We accept the null hypotheses if the $p$-value is greater than the significant level, and we reject it if the $p$-value is smaller than or equal to the significance level. The test appears as a method of testing the long memory parameters. We can perform the properties of this test to estimate parameters using a Monte Carlo simulation which provides the mean, bias and RMSE for a suitable number of replications.

\section{Monte Carlo Experiment}

In this section we carry out the Monte Carlo experiments for several models derived from (1) using different sample sizes with replications.

Under $\left(H_{0}\right)$, we simulate different models using first a strong Gaussian white noise $\varepsilon_{t}$ with mean zero and unit variance and second a GARCH $(1,1)$ noise. 
In that latter case, $\varepsilon_{t}=\sqrt{h_{t}} \xi_{t}, h_{t}=a_{0}+a_{1} \varepsilon_{t-1}^{2}+b_{1} h_{t-1}$, with $\xi_{t}$ a sequence of i.i.d. Gaussian random variables with zero mean and unit variance, $a_{0}=1$, $a_{1}=0.15$ and $b_{1}=0.8$.

We consider 9 models: a model (2), two models (3), two models (5) and four models (6). For the models (3), we use $s=4$ and $s=12$, then we mix the possible explosion at frequency zero with the explosions with fixed seasonalities assuming $s=4$ and $s=12$. For the Gegenbauer models, we detail the results with respect to the location frequency and the number of explosions inside the spectral density. When we have only one factor in the model, the true value of the long memory parameter is $d=0.3$; in presence of two factors, we use $d_{1}=0.3$ and $d_{2}=0.4$; in presence of three factors, we use $d_{1}=0.2, d_{2}=0.3$ and $d_{3}=0.4$.

We consider several sample sizes $T$ from 100 to 3000 . We do not give the results up to 3000 because we intend to apply the method to macro-economic data sets whose sizes are generally smaller. In all cases, we use 2 sizes of replication, $T T=100,1000$. we only present the results for $T T=100$, because the results are quite similar with $T T=1000$. The results are available under request.

We carry out the code on the computer Mac OS X 10.5.1 Léopard, written in language $\mathrm{R}$. The random numbers are generated by the command "rnorm()" as the pseudo random numbers. In the tables the notation $\hat{d}$ represents the mean of the $T T$ " $d$ "s possessing the greatest $p$-value of the test. In the tables, $n$ represents the percentage of times that we get the true value for all the long memory parameters which appear int the models.

We find that for the models with only one term, like models (2) and (6) with $k=1$, the test performs correctly for sample size greater than 900. However, for the models (3), although there is only one parameter to test, the test has no good performance. The performance becomes correct for sample sizes equal to 2000 and 3000 . The same results are observed when we simulate models with several factors, like the models (5) and (6) with $k \geq 2$. The more the explosions are inside the spectral density, the worse is the test's performance. We never got convergence for the test applying at the model (7) as soon as $k>3$. From a general point of view, the performance of the test increases with the sample size.

In summary the results are the following: 
1. First, we assume that the noise $\left(\varepsilon_{t}\right)_{t}$ is a strong white noise in all the models:

- For the model (2), $\hat{d}=0.3$ when $T$ reaches 3000 .

- For the Hassler models, $\hat{d}=0.3$, for $s=4$ when $T$ reaches 3000 , and the test does not converge when we use $s=12$.

- For the models (5), $\hat{d}=0.3$, for $s=4$ and $s=12$, when $T$ reaches 3000 .

- For the 1-factor model (6),

(a) If $\nu=-1, \hat{d}=0.3$ when $T$ reaches 900 .

(b) If $\nu=\cos (\pi / 3), \hat{d}=0.3$ when $T$ reaches 2000 .

- For a 2-factors model (6), $\hat{d}_{1}=0.3$ and $\hat{d}_{2}=0.4$ when $T$ reaches 3000 .

- For a 3-factors model (6), $\hat{d}_{1}=0.2, \hat{d}_{2}=0.3$ and $\hat{d}_{3}=0.4$ when $T$ reaches 2000.

2. Second, we assume that the noise $\left(\varepsilon_{t}\right)_{t}$ is a $\operatorname{GARCH}(1,1)$ noise for all the models:

- For the model (2), $\hat{d}=0.299$ when $T$ reaches 3000 .

- For the model (5), $\hat{d}=0.3$, for $s=4$ when $T$ reaches 3000 , and does not converge when we use $s=12$.

- For the models (5), the test does not converge.

- For the 1-factor model (6),

(a) If $\nu=-1, \hat{d}=0.3$ when $T$ reaches 1000 .

(b) If $\nu=\cos (\pi / 3), \hat{d}=0.3$ when $T$ reaches 2000 .

- For a 2-factors model (6), $\hat{d}_{1}=0.3$ and $\hat{d}_{2}=0.4$ when $T$ reaches 3000 .

- For a 3 -factors model $(6), \hat{d}_{1}=0.2, \hat{d}_{2}=0.3$ and $\hat{d}_{3}=0.4$ when $T$ reaches 3000 .

In the presence of an infinite cycle, comparing the performance of the test when we simulate the models (4) and (5) shows that the convergence is slower. Sometimes the test does not converge at all. We observe also that the test does not converge when we use the Hassler model with $s=12$. In any case, the test convergence is very slow for all the models we use. As soon as we have more than one explosion, we need to use almost 1000 data to be sure to attain in mean the correct estimated value. When we have more than 
two explosions inside the spectral density, it appears difficult to use the test for samples whose size is smaller than 3000. The results are quite similar whatever the noise we use for simulations: a strong white noise or a GARCH noise.

\section{Conclusion}

In this paper, we have evaluated the performance of the tests of Robinson for several simulated SCLM models. We show that the sample size is crucial for the accuracy of the test. It appears that the use of this test is mainly recommended when we observe only one explosion in the spectral density if we have at least 500 points. If several explosions are present inside the periodogram, this test does not provide accurate information if the sample size of the data set is less than 3000. The latter remark raises concern on the application of Robinson test on seasonal macroeconomics data .

\section{References}

[1] Arteche J., Robinson P.M. (2000) Semiparametric inference in seasonal and cyclical long memory processes, Journal of Time Series Analysis, 21, $1-25$.

[2] Arteche J. (2003) Semi-parametric robust tests on seasonal or cyclical long memory time series, Journal of Time Series Analysis, 23, 251 - 285.

[3] Baillie R.T., Bollerslev T., Mikkelsen H.O. (1996), Fractionally integrated generalized autoregressive conditional heteroscedasticity, Journal of Econometrics, 74, 3-30.

[4] Carlin, J.B., Dempster A.P. (1989) Sensitivity analysis of seasonal adjustements: Empirical cas studies, Journal of the American Statistical Association, 84, $6-20$.

[5] Darné O., Guiraud V., Terraza M. (2004) Forecast of the seasonal fractional integrated series, Journal of Forecasting, 23, 1 - 17.

[6] Diongue A. K., Guégan D. (2004) Forecasting electricity spot market prices with a k-factor GIGARCH process, Note de recherché MORA IDHE - nř 9-2004, Décembre 2004, Cachan, France. 
[7] Ferrara, L. and D. Guégan (2000) Forecasting financial time series with generalized long memory processes, in Advances in Quantitative Asset Management, p.319-342, C.L. Dunis eds., Kluwer Academic Publishers.

[8] Ferrara, L. and Guégan, D. (2001a), Forecasting with $k$-factor Gegenbauer processes: Theory and applications, Journal of Forecasting, 20, 581601.

[9] Ferrara, L. and D. Guégan (2001b), Comparison of parameter estimation methods in cyclical long memory time series, in Developments in Forecast Combination and Portfolio Choice, Chapter 8, C. Dunis, J. Moody and A. Timmermann (eds.), Wiley, New York.

[10] Franses P.H., Ooms M. (1997) A periodic long memory model for quartely UK inflation, International Journal of Forecasting, 13, 117 - 126.

[11] Gil-Alana L. A., 2001, A fractionally integrated exponential spectral model for the UK unemployement, J. of Forecasting, 20, 329 -340.

[12] Gil-Alana L. A., 2006, Testing seasonality in the contrext of fractionally integrated processes, Annales d'Economie et de Statistique, 81, 69 - 91.

[13] Giraitis, L., Leipus R. (1995) A generalized Fractionally Differencing Approacg in Long Memory Modelling, Lithuanian Mathematical Journal, $35,65-81$.

[14] Granger C.W.J. and R. Joyeux (1980) An introduction to long memory time series models and fractional differencing, Journal of Time Series Analysis, 1, 15 - 29.

[15] Hosking J.R.M., (1981), Fractional differencing, Biometrika, 68, 1, 165176.

[16] Guégan, D. (2000), A new model : the k-factor GIGARCH process, Journal of Signal Processing, 4, 265-271.

[17] Guégan, D. (2003, A prospective study of the k-factor Gegenbauer process with heteroscedastic errors and an application to inflation rates, $\mathrm{Fi}$ nance India, 17, 1 - 21.

[18] Hassler U. (1994) Misspecification of long memory seasonal time series, Journal of Time Series Analysis, 15, 19 - 30. 
[19] Porter-Hudak, S. (1990) An application to the seasonally fractionally differenced model to the monetary aggregates, Journal of the American Statistical Association, 85, 338 - 344.

[20] Ray B.K. (1993) Long-range forecasting of IBM product revenues using a seasonal fractionally differenced ARMA model, International Journal of Forecasting, 9, 255 - 269.

[5] Robinson, P.M., 1994. Efficient tests of nonstationary hypotheses. J. of the American Statistical Association, 89 (428), 1420-1437.

[21] Woodward, W.A., Cheng Q.C. and Gray H.L. (1998) A $k$-factor GARMA long-memory model, Journal of Time Series Analysis, 19, 5, 485-504. 


\begin{tabular}{|c|c|c|c|c|c|c|c|c|}
\hline $\mathrm{T}$ & 100 & 300 & 500 & 700 & 900 & 1000 & 2000 & 3000 \\
\hline $\mathrm{n}$ & 37 & 67 & 80 & 88 & 94 & 98 & 100 & 100 \\
\hline$\hat{d}$ & 0.248 & 0.283 & 0.288 & 0.29 & 0.302 & 0.3 & 0.3 & 0.3 \\
\hline
\end{tabular}

Table 1: Test for model $(1-B)^{0.3} X_{t}=\varepsilon_{t}$ where $\varepsilon_{t} \sim \mathrm{N}(0,1)$.

\begin{tabular}{|c|c|c|c|c|c|c|c|c|}
\hline $\mathrm{T}$ & 100 & 300 & 500 & 700 & 900 & 1000 & 2000 & 3000 \\
\hline $\mathrm{n}$ & 7 & 43 & 58 & 72 & 85 & 92 & 99 & 100 \\
\hline$\hat{d}$ & 0.092 & 0.235 & 0.258 & 0.276 & 0.285 & 0.292 & 0.299 & 0.3 \\
\hline
\end{tabular}

Table 2: Test for model $\left(1-B^{4}\right)^{0.3} X_{t}=\varepsilon_{t}$ where $\varepsilon_{t} \sim \mathrm{N}(0,1)$.

\begin{tabular}{|c|c|c|c|c|c|c|c|c|}
\hline $\mathrm{T}$ & 100 & 300 & 500 & 700 & 900 & 1000 & 2000 & 3000 \\
\hline $\mathrm{n}$ & 0 & 0 & 3 & 4 & 18 & 26 & 78 & 98 \\
\hline$\hat{d}$ & 0.0 & 0.05 & 0.148 & 0.191 & 0.217 & 0.2223 & 0.278 & 0.298 \\
\hline
\end{tabular}

Table 3: Test for model $\left(1-B^{12}\right)^{0.3} X_{t}=\varepsilon_{t}$ where $\varepsilon_{t} \sim \mathrm{N}(0,1)$.

\begin{tabular}{|c|c|c|c|c|c|c|c|c|}
\hline $\mathrm{T}$ & 100 & 300 & 500 & 700 & 900 & 1000 & 2000 & 3000 \\
\hline $\mathrm{n}$ & 3 & 17 & 28 & 50 & 51 & 63 & 87 & 92 \\
\hline$\hat{d}_{1}$ & 0.279 & 0.296 & 0.305 & 0.299 & 0.299 & 0.3 & 0.3 & 0.302 \\
\hline$\hat{d}_{2}$ & 0.154 & 0.332 & 0.346 & 0.372 & 0.377 & 0.386 & 0.396 & 0.399 \\
\hline
\end{tabular}

Table 4: Test for model $(1-B)^{0.3}\left(1-B^{4}\right)^{0.4} X_{t}=\varepsilon_{t}$ where $\varepsilon_{t} \sim \mathrm{N}(0,1)$.

\begin{tabular}{|c|c|c|c|c|c|c|c|c|}
\hline $\mathrm{T}$ & 100 & 300 & 500 & 700 & 900 & 1000 & 2000 & 3000 \\
\hline $\mathrm{n}$ & 0 & 3 & 4 & 10 & 20 & 27 & 71 & 92 \\
\hline$\hat{d}_{1}$ & 0.299 & 0.304 & 0.304 & 0.298 & 0.302 & 0.307 & 0.3 & 0.301 \\
\hline$\hat{d}_{2}$ & 0.001 & 0.137 & 0.245 & 0.284 & 0.322 & 0.329 & 0.373 & 0.394 \\
\hline
\end{tabular}

Table 5: Test for model $(1-B)^{0.3}\left(1-B^{12}\right)^{0.4} X_{t}=\varepsilon_{t}$ where $\varepsilon_{t} \sim \mathrm{N}(0,1)$.

\begin{tabular}{|c|c|c|c|c|c|c|c|c|}
\hline $\mathrm{T}$ & 100 & 300 & 500 & 700 & 900 & 1000 & 2000 & 3000 \\
\hline $\mathrm{n}$ & 34 & 65 & 78 & 89 & 94 & 98 & 100 & 100 \\
\hline$\hat{d}$ & 0.246 & 0.285 & 0.286 & 0.297 & 0.3 & 0.3 & 0.3 & 0.3 \\
\hline
\end{tabular}

Table 6: Test for model $\left(1-2 \nu B+B^{2}\right)^{0.15} X_{t}=(1+B)^{0.3}=\varepsilon_{t}$ where $\varepsilon_{t} \sim \mathrm{N}(0,1)$. 


\begin{tabular}{|c|c|c|c|c|c|c|c|c|}
\hline $\mathrm{T}$ & 100 & 300 & 500 & 700 & 900 & 1000 & 2000 & 3000 \\
\hline $\mathrm{n}$ & 18 & 60 & 78 & 94 & 98 & 95 & 100 & 100 \\
\hline$\hat{d}$ & 0.171 & 0.266 & 0.278 & 0.294 & 0.298 & 0.297 & 0.3 & 0.3 \\
\hline
\end{tabular}

Table 7: Test for model $\left(1-2 \nu B+B^{2}\right)^{0.3} X_{t}=\varepsilon_{t}, \nu=\cos \frac{\pi}{3}$ and $\varepsilon_{t} \sim \mathrm{N}(0,1)$.

\begin{tabular}{|c|c|c|c|c|c|c|c|c|}
\hline $\mathrm{T}$ & 100 & 300 & 500 & 700 & 900 & 1000 & 2000 & 3000 \\
\hline $\mathrm{n}$ & 0 & 23 & 32 & 52 & 69 & 67 & 94 & 100 \\
\hline$\hat{d}_{1}$ & 0.068 & 0.192 & 0.231 & 0.254 & 0.277 & 0.28 & 0.295 & 0.3 \\
\hline$\hat{d}_{2}$ & 0.223 & 0.322 & 0.351 & 0.372 & 0.388 & 0.389 & 0.398 & 0.4 \\
\hline
\end{tabular}

Table 8: Test for model $\left(1-2 \nu_{1} B+B^{2}\right)^{0.3}\left(1-2 \nu_{2} B+B^{2}\right)^{0.4} X_{t}=\varepsilon_{t}, \nu_{1}=\cos \frac{\pi}{3}, \nu_{2}=$ $\cos \frac{5 \pi}{6}$ and $\varepsilon_{t} \sim \mathrm{N}(0,1)$.

\begin{tabular}{|c|c|c|c|c|c|c|c|c|}
\hline $\mathrm{T}$ & 100 & 300 & 500 & 700 & 900 & 1000 & 2000 & 3000 \\
\hline $\mathrm{n}$ & 0 & 8 & 33 & 58 & 77 & 73 & 100 & 100 \\
\hline$\hat{d}_{1}$ & 0.023 & 0.129 & 0.156 & 0.176 & 0.192 & 0.187 & 0.2 & 0.2 \\
\hline$\hat{d}_{2}$ & 0.16 & 0.245 & 0.271 & 0.284 & 0.295 & 0.286 & 0.3 & 0.3 \\
\hline$\hat{d}_{3}$ & 0.133 & 0.306 & 0.353 & 0.369 & 0.388 & 0.385 & 0.4 & 0.4 \\
\hline
\end{tabular}

Table 9: Test for model $\left(1-2 \nu_{1} B+B^{2}\right)^{0.2}\left(1-2 \nu_{2} B+B^{2}\right)^{0.3}\left(1-2 \nu_{3} B+B^{2}\right)^{0.4} X_{t}=\varepsilon_{t}$, $\nu_{1}=\cos \frac{\pi}{6}, \nu_{2}=\cos \frac{\pi}{2}, \nu_{3}=\cos \frac{2 \pi}{3}$ and $\varepsilon_{t} \sim \mathrm{N}(0,1)$.

\begin{tabular}{|c|c|c|c|c|c|c|c|c|}
\hline $\mathrm{T}$ & 100 & 300 & 500 & 700 & 900 & 1000 & 2000 & 3000 \\
\hline $\mathrm{n}$ & 34 & 56 & 72 & 80 & 83 & 84 & 99 & 99 \\
\hline$\hat{d}$ & 0.249 & 0.279 & 0.292 & 0.296 & 0.301 & 0.298 & 0.299 & 0.299 \\
\hline
\end{tabular}

Table 10: Test for model $(1-B)^{0.3} X_{t}=\varepsilon_{t}$ where $\varepsilon_{t} \sim \operatorname{GARCH}(1,1)$.

\begin{tabular}{|c|c|c|c|c|c|c|c|c|}
\hline $\mathrm{T}$ & 100 & 300 & 500 & 700 & 900 & 1000 & 2000 & 3000 \\
\hline $\mathrm{n}$ & 6 & 38 & 43 & 64 & 81 & 72 & 91 & 98 \\
\hline$\hat{d}$ & 0.112 & 0.235 & 0.244 & 0.27 & 0.281 & 0.272 & 0.291 & 0.3 \\
\hline
\end{tabular}

Table 11: Test for model $\left(1-B^{4}\right)^{0.3} X_{t}=\varepsilon_{t}$ where $\varepsilon_{t} \sim \operatorname{GARCH}(1,1)$.

\begin{tabular}{|c|c|c|c|c|c|c|c|c|}
\hline $\mathrm{T}$ & 100 & 300 & 500 & 700 & 900 & 1000 & 2000 & 3000 \\
\hline $\mathrm{n}$ & 0 & 1 & 7 & 12 & 20 & 29 & 75 & 94 \\
\hline$\hat{d}$ & 0.0 & 0.06 & 0.156 & 0.192 & 0.218 & 0.229 & 0.275 & 0.294 \\
\hline
\end{tabular}

Table 12: Test for model $\left(1-B^{12}\right)^{0.3} X_{t}=\varepsilon_{t}$ where $\varepsilon_{t} \sim \operatorname{GARCH}(1,1)$. 


\begin{tabular}{|c|c|c|c|c|c|c|c|c|}
\hline $\mathrm{T}$ & 100 & 300 & 500 & 700 & 900 & 1000 & 2000 & 3000 \\
\hline $\mathrm{n}$ & 0 & 14 & 22 & 36 & 50 & 54 & 83 & 86 \\
\hline$\hat{d}_{1}$ & 0.283 & 0.297 & 0.312 & 0.304 & 0.3 & 0.298 & 0.306 & 0.299 \\
\hline$\hat{d}_{2}$ & 0.152 & 0.319 & 0.34 & 0.372 & 0.381 & 0.385 & 0.393 & 0.395 \\
\hline
\end{tabular}

Table 13: Test for model $(1-B)^{0.3}\left(1-B^{4}\right)^{0.4} X_{t}=\varepsilon_{t}$ where $\varepsilon_{t} \sim \operatorname{GARCH}(1,1)$.

\begin{tabular}{|c|c|c|c|c|c|c|c|c|}
\hline $\mathrm{T}$ & 100 & 300 & 500 & 700 & 900 & 1000 & 2000 & 3000 \\
\hline $\mathrm{n}$ & 0 & 0 & 5 & 8 & 22 & 20 & 63 & 81 \\
\hline$\hat{d}_{1}$ & 0.294 & 0.28 & 0.298 & 0.299 & 0.303 & 0.305 & 0.304 & 0.304 \\
\hline$\hat{d}_{2}$ & 0 & 0.117 & 0.249 & 0.285 & 0.314 & 0.323 & 0.37 & 0.386 \\
\hline
\end{tabular}

Table 14: Test for model $(1-B)^{0.3}\left(1-B^{12}\right)^{0.4} X_{t}=\varepsilon_{t}$ where $\varepsilon_{t} \sim \operatorname{GARCH}(1,1)$.

\begin{tabular}{|c|c|c|c|c|c|c|c|c|}
\hline $\mathrm{T}$ & 100 & 300 & 500 & 700 & 900 & 1000 & 2000 & 3000 \\
\hline $\mathrm{n}$ & 31 & 59 & 71 & 84 & 86 & 86 & 94 & 100 \\
\hline$\hat{d}$ & 0.232 & 0.294 & 0.291 & 0.304 & 0.299 & 0.3 & 0.3 & 0.3 \\
\hline
\end{tabular}

Table 15: Test for model $\left(1-2 \nu B+B^{2}\right)^{0.15} X_{t}=(1+B)^{0.3}=\varepsilon_{t}$ where $\varepsilon_{t} \sim \operatorname{GARCH}(1,1)$.

\begin{tabular}{|c|c|c|c|c|c|c|c|c|}
\hline $\mathrm{T}$ & 100 & 300 & 500 & 700 & 900 & 1000 & 2000 & 3000 \\
\hline $\mathrm{n}$ & 20 & 57 & 82 & 79 & 84 & 89 & 100 & 100 \\
\hline$\hat{d}$ & 0.181 & 0.269 & 0.283 & 0.289 & 0.29 & 0.293 & 0.3 & 0.3 \\
\hline
\end{tabular}

Table 16: Test for model $\left(1-2 \nu B+B^{2}\right)^{0.3} X_{t}=\varepsilon_{t}, \nu=\cos \frac{\pi}{3}$ and $\varepsilon_{t} \sim \operatorname{GARCH}(1,1)$.

\begin{tabular}{|c|c|c|c|c|c|c|c|c|}
\hline $\mathrm{T}$ & 100 & 300 & 500 & 700 & 900 & 1000 & 2000 & 3000 \\
\hline $\mathrm{n}$ & 2 & 17 & 39 & 51 & 50 & 63 & 85 & 100 \\
\hline$\hat{d}_{1}$ & 0.062 & 0.196 & 0.249 & 0.25 & 0.266 & 0.272 & 0.3 & 0.3 \\
\hline$\hat{d}_{2}$ & 0.218 & 0.331 & 0.362 & 0.367 & 0.374 & 0.383 & 0.394 & 0.4 \\
\hline
\end{tabular}

Table 17: Test for model $\left(1-2 \nu_{1} B+B^{2}\right)^{0.3}\left(1-2 \nu_{2} B+B^{2}\right)^{0.4} X_{t}=\varepsilon_{t}, \nu_{1}=\cos \frac{\pi}{3}, \nu_{2}=$ $\cos \frac{5 \pi}{6}$ and $\varepsilon_{t} \sim \operatorname{GARCH}(1,1)$.

\begin{tabular}{|c|c|c|c|c|c|c|c|c|}
\hline $\mathrm{T}$ & 100 & 300 & 500 & 700 & 900 & 1000 & 2000 & 3000 \\
\hline $\mathrm{n}$ & 0 & 10 & 23 & 38 & 62 & 58 & 95 & 97 \\
\hline$\hat{d}_{1}$ & 0.023 & 0.138 & 0.156 & 0.172 & 0.187 & 0.177 & 0.198 & 0.195 \\
\hline$\hat{d}_{2}$ & 0.167 & 0.249 & 0.281 & 0.277 & 0.284 & 0.283 & 0.302 & 0.3 \\
\hline$\hat{d}_{3}$ & 0.161 & 0.328 & 0.346 & 0.365 & 0.384 & 0.378 & 0.397 & 0.4 \\
\hline
\end{tabular}

Table 18: Test for model $\left(1-2 \nu_{1} B+B^{2}\right)^{0.2}\left(1-2 \nu_{2} B+B^{2}\right)^{0.3}\left(1-2 \nu_{3} B+B^{2}\right)^{0.4} X_{t}=\varepsilon_{t}$, $\nu_{1}=\cos \frac{\pi}{6}, \nu_{2}=\cos \frac{\pi}{2}, \nu_{3}=\cos \frac{2 \pi}{3}$ and $\varepsilon_{t} \sim \operatorname{GARCH}(1,1)$. 